\title{
Low-dose testosterone alleviates vascular damage caused by castration in male rats in puberty via modulation of the PI3K/AKT signaling pathway
}

\author{
JING ZHAO ${ }^{1,2}$, GE-LI LIU ${ }^{1}$, YING WEI ${ }^{1}$, LI-HONG JIANG ${ }^{1}$, PENG-LI BAO ${ }^{1}$ and QING-YAN YANG ${ }^{1}$ \\ ${ }^{1}$ Department of Paediatrics, General Hospital of Tianjin Medical University, Tianjin 300052; \\ ${ }^{2}$ Department of Neonatology, Tianjin Central Hospital of Gynecology Obstetrics, Tianjin 415400, P.R. China
}

Received March 4, 2015; Accepted February 2, 2016

DOI: $10.3892 / \mathrm{mmr} .2016 .5562$

\begin{abstract}
The aim of the present study was to investigate the effect of testosterone on glucolipid metabolism and vascular injury in male rats, and examine the underlying molecular mechanisms. A total of 40 male Sprague-Dawley rats were divided into a control group $(n=10)$, high-fat-diet + castration group $(n=10)$, high-fat-diet + castration + low dose testosterone group $(n=10)$, and high-fat-diet + castration + high dose testosterone group $(n=10)$. Hematoxylin and eosin staining was performed to evaluate the morphology of the thoracic aortic tissues. Immunohistochemical staining was used to detect biomarkers of the phosphoinositide 3-kinase (PI3K) signaling pathway. The mRNA and protein expression levels of PI3K, AKT, insulin receptor substrate-1 (IRS-1), glucose transporter type 4 (GLUT-4), nuclear factor (NF)- $\kappa$ B and tumor necrosis factor (TNF)- $\alpha$ in the aortas were determined using quantitative polymerase chain reaction and Western blot analyses, respectively. Apoptosis in the aortic tissues was detected using a TUNEL assay. Castration induced apoptosis in the animals fed a high-fat-diet, whereas low dose testosterone replacement ameliorated the apoptosis in the aorta. However, the levels of apoptosis was more severe following high-dose testosterone treatment. Low-dose testosterone induced upregulation in the levels of IRS-1, AKT, GLUT-4 protein, NF- $\kappa$ B, TNF- $\alpha$ and PI3K, compared with those in the animals fed a high-fat diet following castration. A high dose of testosterone resulted in a significant decrease in the levels of IRS-1, AKT, GLUT-4, NF- $\kappa \mathrm{B}, \mathrm{TNF}-\alpha$ and PI3K. Compared with the rats in the high-fat diet + castration group, a low dose of testosterone induced upregulation in the mRNA levels of IRS-1, AKT and GLUT-4, and downregulation of the mRNA levels of $N F-\kappa B$,
\end{abstract}

Correspondence to: Professor Ge-Li Liu, Department of Paediatrics, General Hospital of Tianjin Medical University, 154 Anshan Road, Heping, Tianjin 300052, P.R. China

E-mail: liugeli008@sina.com

Key words: testosterone, castration, puberty, phosphoinositide 3-kinase/AKT signaling pathway, obesity, vascular injuries
TNF- $\alpha$ and PI3K. A high dose of testosterone resulted in a significant decrease in the levels of IRS-1, AKT and GLUT-4, and marked increases in the mRNA levels of NF- $\kappa \mathrm{B}, \mathrm{TNF}-\alpha$ and PI $3 \mathrm{~K}$, compared with the low dose group. Castration induced marked disorders of glucolipid metabolism and vascular injuries in the pubescent male rats. Low-dose testosterone treatment was found to ameliorate the vascular damage caused by castration via the PI3K/AKT signaling pathway.

\section{Introduction}

Improvements in quality of living and changes in diet have been identified as the major issues contributing to the escalation of obesity in children and adolescents (1). To date, obesity is considered to be associated with insulin resistance, abnormal glucose metabolism, dyslipidemia, inflammation and vascular damage (2). The increasing prevalence of peripubertal obesity has raised the concerns regarding the prevalence and severity of adolescent metabolic syndrome $(3,4)$.

Epidemiological data indicates a higher incidence of diabetes mellitus and fatty liver in males than in females, which may be associated with the expression of endogenous sex hormones. In males, testosterone is important in the body composition, particularly of visceral fat (5). For example, increases in serum triglyceride and total cholesterol (TC) have been observed in men with testosterone deficiency (6). In addition, decreased plasma testosterone has been identified in obese adolescent males, compared with control individuals of the same Tanner stage, consistent with data in adult males, associating obesity and insulin resistance with hypotestosteronemia $(7,8)$. Furthermore, testosterone replacement treatment can enhance the effect of insulin and contribute to glucolipid metabolism, whereas high doses of exogenous testosterone decrease insulin sensitivity $(9,10)$.

Increasing attention has been focused on the association between androgen deficiency and a variety of cardiovascular diseases $(11,12)$. For example, a decrease in endogenous testosterone is a risk factor for atherosclerosis in males (11). In addition, short-term administration of testosterone at physiological concentrations can ameliorate coronary heart disease and improve endothelial vascular function (13). To date, the roles of testosterone in the metabolism of glucolipids and cardiovascular 
disease in obesity remain to be fully elucidated. In the present study, a male rat model subjected to a high-fat diet and castration was established, based on which the present study aimed to examine the effect of testosterone in these animals. The results may determine whether testosterone attenuates vascular injury and assist in elucidating the underlying mechanism.

\section{Materials and methods}

Animals. For the animal model, 40 male Sprague-Dawley rats (3-week-old, weighing 50-60 g) were provided by the Academy of Military Medical Sciences (Beijing, China). All rats were housed at a temperature of $26^{\circ} \mathrm{C}$ in a $12 \mathrm{~h}$ light $/ 12 \mathrm{~h}$ dark cycle, and were provided with free access to water and food. Following adaptive breeding for 1 week, the animals were randomly divided into the following groups: Group 1, control group $(n=10)$, in which rats were fed a high-fat diet containing $57 \%$ carbohydrate, $22 \%$ protein and 4\% fat; group 2, high-fat-diet + castration group $(n=10)$, which were fed a high-fat diet following castration; group 3, high-fat-diet + castration + low dose testosterone group $(\mathrm{n}=10)$, which were fed a high-fat diet following castration, and were administered with testosterone (Sigma-Aldrich, St. Louis, MO, USA) at a dose of $12.5 \mu \mathrm{g} / \mathrm{kg} /$ day (dissolved in sesame oil); group 4, high-fat-diet + castration + high dose testosterone group $(\mathrm{n}=10)$, which were fed a high-fat diet following castration, and received testosterone at a dose of $25 \mu \mathrm{g} / \mathrm{kg} /$ day. Castration was performed by removal of the testicles following anesthesia using $10 \%$ chloral hydrate $(3.5 \mathrm{ml} / \mathrm{kg}$; Beijing Solarbio Science \& Technology Co., Ltd., Beijing, China) via intraperitoneal injection. All animals were fed for 6 weeks, and body weights were monitored weekly. The rats were sacrificed by cervical dislocation at the age of 10 weeks, and the thoracic aortas were obtained for subsequent experiments. All experimental protocols were approved by the Ethics Committee of the General Hospital of Tianjin Medical University (Tianjin, China).

Biochemical measurements. Prior to blood collection, the rats were maintained in a fasting state for $24 \mathrm{~h}$. Blood samples (3-5 ml) were collected from an angular vein, followed by centrifugation at $1,680 \mathrm{x} \mathrm{g}$ and $4^{\circ} \mathrm{C}$ for $15 \mathrm{~min}$. Subsequently, the levels of $\mathrm{TC}$, high-density lipoprotein cholesterol (HDL-C), insulin, glucose and testosterone in the serum were determined using an Olympus AU400 Clinical Chemistry Analyzer (Olympus Coporation, Tokyo, Japan). Non-HDL cholesterol was defined as total cholesterol - HDL cholesterol. Serum glucose was determined using a glucose oxidase kit purchased from Biosino Bio-Technology \& Science Inc. (Beijing, China). Serum insulin was measured using a rat Insulin ELISA kit purchased from the China Institute of Atomic Energy (Beijing, China). Plasma lipids were measured using Hitachi 7170 automatic biochemistry analyzer (Hitachi, Tokyo, Japan). Homeostatic model assessment-insulin resistance (HOMA-IR) was calculated according to the following formula: HOMA-IR = fasting insulin $(\mu \mathrm{U} / \mathrm{ml}) \times$ fasting serum glucose $(\mathrm{mmol} / \mathrm{l}) / 22.5$.

Immunohistochemical staining. For immunohistochemical analyses, the paraffin-embedded sections $(8 \mu \mathrm{m})$ were dewaxed for $10 \mathrm{~min}$, followed by immersion in boiling citrate buffer solution (pH 6.4; Beijing Solarbio Science \& Technology Co.,Ltd.) for
Table I. Primers used for reverse transcription-quantitative polymerase chain reaction analysis.

\begin{tabular}{|c|c|}
\hline Gene & Primer sequence $\left(5^{\prime}-3^{\prime}\right)$ \\
\hline $\mathrm{NF}-\kappa \mathrm{B}$ & $\begin{array}{l}\text { Forward: TGAGGCTGTTTGGTTTGAGA } \\
\text { Reverse:TTATGGCTGAGGTCTGGTCTG }\end{array}$ \\
\hline TNF- $\alpha$ & $\begin{array}{l}\text { Forward: GCTCCCTCTCATCAGTTCCA } \\
\text { Reverse: GCTTGGTGGTTTGCTACGAC }\end{array}$ \\
\hline GLUT-4 & $\begin{array}{l}\text { Forward: GTATGTTGCGGATGCTATGG } \\
\text { Reverse: CCTCTGGTTTCAGGCACTCT }\end{array}$ \\
\hline IRS-1 & $\begin{array}{l}\text { Forward: GGCACCATCTCAACAATCCT } \\
\text { Reverse: GTTTCCCACCCACCATACTG }\end{array}$ \\
\hline PI3K & $\begin{array}{l}\text { Forward: CAAAGCCGAGAACCTATTGC } \\
\text { Reverse: TTGACTTCGCCATCTACCAC }\end{array}$ \\
\hline $\mathrm{AKT}$ & $\begin{array}{l}\text { Forward: TGGCACCTTTATTGGCTACA } \\
\text { Reverse: CCGCTCTGTCTTCATCAGC }\end{array}$ \\
\hline$\beta$-actin & $\begin{array}{l}\text { Forward: CGTTGACATCCGTAAAGACC } \\
\text { Reverse: AGAGCCACCAATCCACACA }\end{array}$ \\
\hline
\end{tabular}

NF- $\kappa \mathrm{B}$, nuclear factor- $\kappa \mathrm{B}$; TNF- $\alpha$, tumor necrosis factor- $\alpha$; GLUT-4; glucose transporter type 4; IRS-1, insulin receptor substrate-1; PI3K, phosphoinositide 3-kinase.

$10 \mathrm{~min}$. Antigen retrieval was performed by microwave heating at a high heat for $10 \mathrm{~min}$, a low heat for $10 \mathrm{~min}$ and cooled to room temperature. The sections were then immersed in distilled water in an orbital shaker for $5 \mathrm{~min}$. Following antigen retrieval, the sections were incubated in $3 \% \mathrm{H}_{2} \mathrm{O}_{2}$ solution (Beijing Solarbio Science \& Technology Co., Ltd.) for $5 \mathrm{~min}$ at room temperature, and then blocked with $5 \%$ bovine serum albumin (Beijing Solarbio Science \& Technology Co., Ltd.) for $1 \mathrm{~h}$ to avoid nonspecific staining. The sections were then incubated with the following primary antibodies: Polyclonal rabbit anti-rat IRS-1 (1:100; Abcam, Cambridge, UK; cat. no. ab131487), polyclonal rabbit anti-rat GLUT-4 (1:400; Abcam; cat. no. ab654), polyclonal rabbit anti-rat TNF- $\alpha$ (1:1,000; Cell Signaling Technology, Inc., Danvers, MA, USA; cat. no. 21926) and polyclonal rabbit anti-rat NF- $\mathrm{B}$ (1:800; Cell Signaling Technology, Inc.; cat. no. 5970) at $4^{\circ} \mathrm{C}$ overnight. Subsequently, the sections were incubated with biotin-conjugated monoclonal goat anti-rabbit IgG (1:200; Vector Laboratories, Inc., Burlingame, CA, USA; cat. no. BA-1000) for $2 \mathrm{~h}$ at room temperature, and horseradish peroxidase (HRP) streptavidin (Vector laboratories, Inc.) for $1 \mathrm{~h}$ at room temperature. The images were analyzed using Image-Pro Plus 6.0 software (Media Cybernetics, Silver Spring, MD, USA).

TUNEL assay. The tissue sections $(5 \mu \mathrm{m})$ obtained from the thoracic aorta were cut on a freezing microtome (Leica RM2255; Leica Microsystems, Wetzlar, Germany). The numbers and distribution of apoptotic cells within the aortic tissues were analyzed using an in situ Cell Death Detection kit (Roche Diagnostics GmbH, Mannheim, Germany) according to the manufacturer's protocol. Subsequently, the sections were incubated with $40 \mu \mathrm{l}$ reaction mixture containing terminal 
A

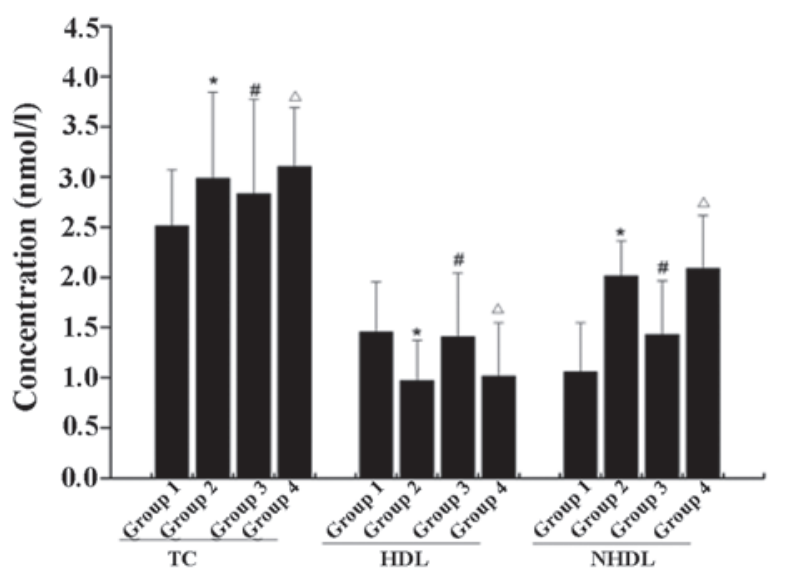

B

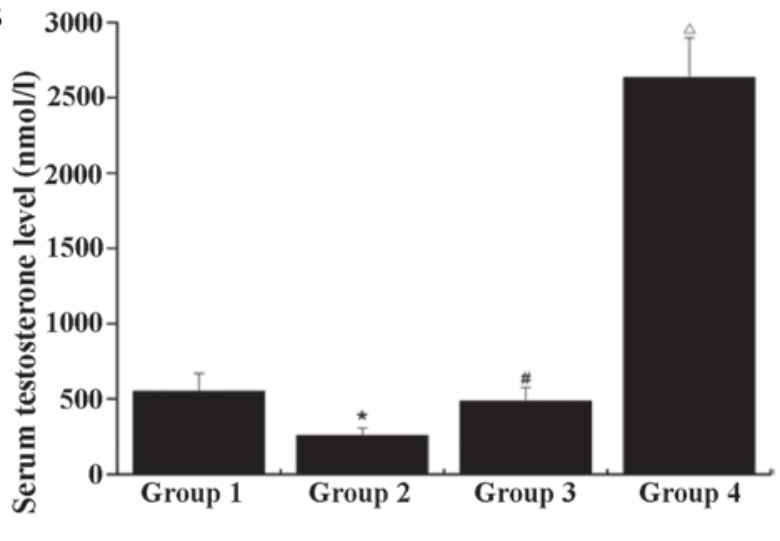

C

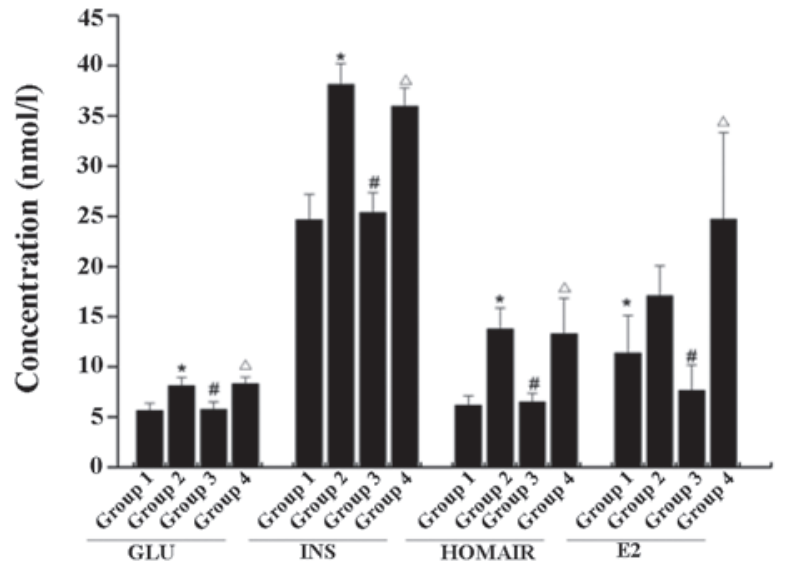

Figure 1. Serum levels of (A) TC, HDL and NHDL, (B) testosterone, (C) GLU, INS, HOMA-IR and E2 in rats. The data are presented as the mean \pm standard deviation, based on three independent experiments. ${ }^{*} \mathrm{P}<0.05$, compared with the control group; ${ }^{\prime \prime} \mathrm{P}<0.05$, group 3 compared with group $2,{ }^{\Delta} \mathrm{P}<0.05$, group 4 compared with group 2. TC, total cholesterol; HDL, high density lipoprotein; NHDL, non-high density lipoprotein; GLU, glucose; INS, insulin; HOMA-IR, homeostatic model assessment-insulin resistance; E2, estradiol.

deoxynucleotidyltransferase (TdT) and digoxigenin-conjugated dUTP for $1 \mathrm{~h}$ at $37^{\circ} \mathrm{C}$. Subsequently, the sections were stained with DAPI (Roche Diagnostics GmbH, Mannheim, Germany) to stain the nuclei for $1 \mathrm{~min}$ at room temperature, followed by washing with phosphate-buffered saline. Finally, the TUNEL (Roche Diagnostics $\mathrm{GmbH}$ )-positive cells were observed under a fluorescent microscope (Olympus BX51TF; Olympus, Tokyo, Japan) at x200 magnification.

Western blot analysis. Protein extraction from the aortic tissues was performed using tissue lysis buffer (Solarbio, Beijing, China) containing 1\% phenylmethanesulfonyl fluoride and protein was quantified using a BCA protein assay kit. The protein $(25 \mu \mathrm{g})$ was separated on a $10 \%$ SDS-PAGE gel (Beijing Solarbio Science \& Technology Co., Ltd.) and transferred onto polyvinylidene fluoride membranes (Merck Millipore, Darmstadt, Germany). Subsequently, the membrane was blocked with $5 \%$ nonfat dried milk for $2 \mathrm{~h}$ at room temperature and then incubated with rabbit primary antibodies against NF- $\mathrm{KB}, \mathrm{TNF}-\alpha$ and polyclonal rabbit anti-rat $\beta$-actin (1:1,000; Cell Signaling Technology, Inc.; cat. no. 4967), GLUT4 and IRS1 (1:2,000; Abcam) and polyclonal rabbit anti-rat PI3K (1:1,000, Sigma-Aldrich; cat. no. 5295) overnight at $4^{\circ} \mathrm{C}$. The membrane was washed with $0.1 \%$ PBST three times for $5 \mathrm{~min}$. Subsequently, the membrane was incubated with HRP-labeled goat anti-rabbit
IgG (cat. no. GGHL-15P; ICL, Inc., Portland, OR, USA) secondary antibodies for $1 \mathrm{~h}$ at room temperature. The same membranes probed for $\beta$-actin served as an internal standard. The relative density of protein to $\beta$-actin was analyzed using ImageJ software (version 1.43; National Institutes of Health, Bethesda, MA, USA).

Reverse transcription-quantitative polymerase chain reaction $(R T-q P C R)$. Total RNA was extracted from the aortic tissues using TRIzol reagent (Invitrogen; Thermo Fisher Scientific, Inc.), according to the manufacturer's protocol. Synthesis of cDNA was performed using a TransScript First-Strand cDNA system (TransGen Biotech, Beijing, China), according to the manufacturer's protocol. The reaction mixture consisted of $10 \mu \mathrm{l}$ of $2 \mathrm{X}$ TransStart Top Green qPCR SuperMIX, $0.4 \mu 1$ forward primer, $0.4 \mu 1$ reverse primer, $0.4 \mu \mathrm{l}$ passive reference dye, $1.0 \mu \mathrm{l}$ cDNA template

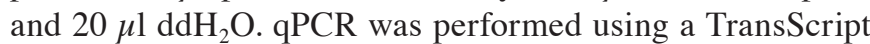
Top Green qPCR SuperMix kit (TransGen Biotech) on a BioRad CFX96 qPCR system (Bio-Rad Laboratories, Inc., Hercules, CA, USA) using the primers listed in Table I. The PCR amplification was conducted using the following conditions: $94^{\circ} \mathrm{C}$ for $30 \mathrm{sec}$, followed by 40 cycles of $94^{\circ} \mathrm{C}$ for $5 \mathrm{sec}, 60^{\circ} \mathrm{C}$ for $15 \mathrm{sec}$ and $72^{\circ} \mathrm{C}$ for $10 \mathrm{sec}$. The relative quantification of each gene was normalized to $\beta$-actin using the $2^{-\Delta \Delta \mathrm{Cq}}$ method (14). 
A

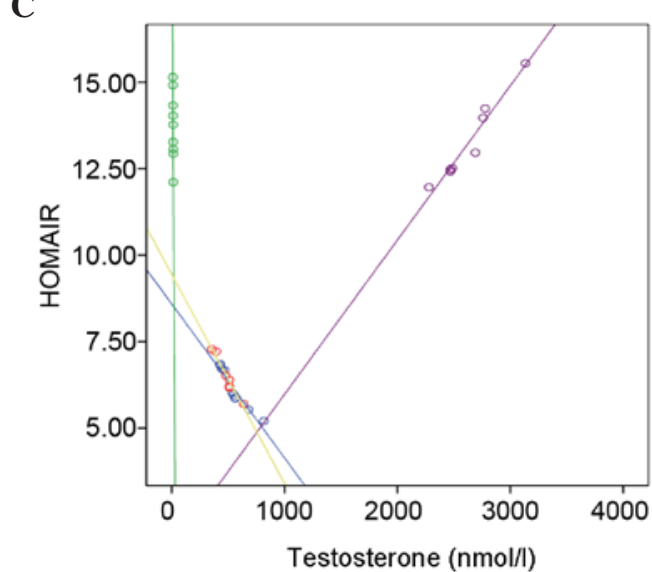

$\mathbf{E}$

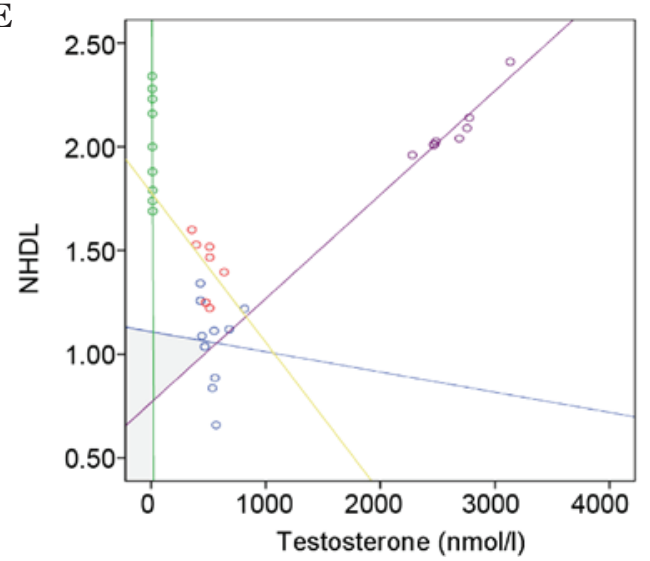

B
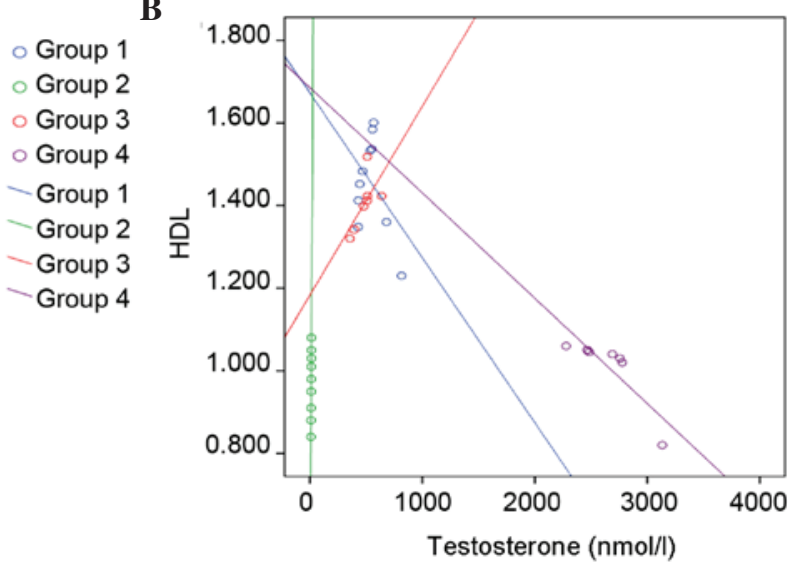

D
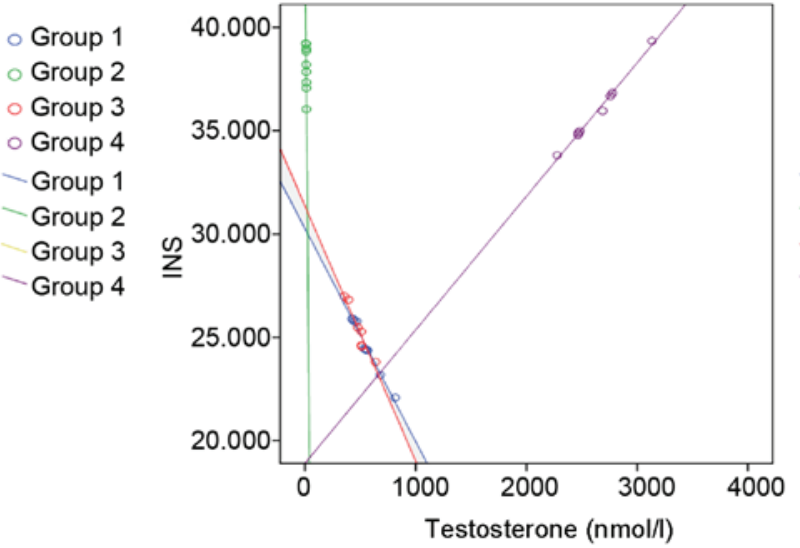

- Group 1

- Group 2

- Group 3

- Group 4

Group 1

Group 2

Group 3

Group 4

\section{$\mathbf{F}$}

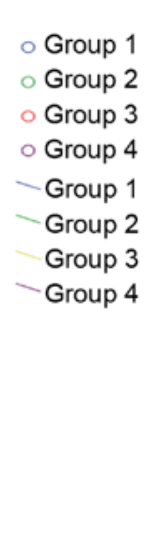

Figure 2. Scatter diagrams of testosterone and metabolism indices of (A) GLU, (B) HDL, (C) HOMRIR, (D) INS, (E) NHDL and (F) TC. HDL, high density lipoprotein; NHDL, non-high density lipoprotein; GLU, glucose; INS, insulin; HOMA-IR, homeostatic model assessment-insulin resistance; TC, total cholesterol.

Statistical analysis. Statistical analysis was performed using SPSS 17 software (SPSS, Inc., Chicago, IL, USA). All data are expressed as the mean \pm standard error of the mean. The inter-group differences were analyzed using analysis of variance. Student's $t$-test was performed to analyze the measurement data. $\mathrm{P}<0.05$ was considered to indicate a statistically significant difference.

\section{Results}

Biochemical parameters. Compared with control (group 1), significant increases were found in the plasma levels of glucose, insulin, HOMA-IR, TC and non-HDL in group $2(\mathrm{P}<0.05$;
Fig. 1). A significant decrease was revealed in the levels of HDL and testosterone in the plasma of group 2, compared with the normal control $(\mathrm{P}<0.05)$. In the animals subjected to low dose testosterone replacement (group 3), significant reductions in plasma TC and non-HDL were observed, compared with group $2(\mathrm{P}<0.05)$. The plasma levels of glucose, insulin, $\mathrm{TC}$ and non-HDL were further increased following exposure to high dose testosterone (group 4), compared with group $2(\mathrm{P}<0.05)$.

In the scatter diagram of testosterone and the metabolism of the associated parameters, a continual decrease was identified in the plasma levels of glucose, insulin, HOMA-IR, TC and non-HDL as plasma testosterone increased in groups 1,2 and 3, respectively (Fig. 2). However, an increasing trend was 


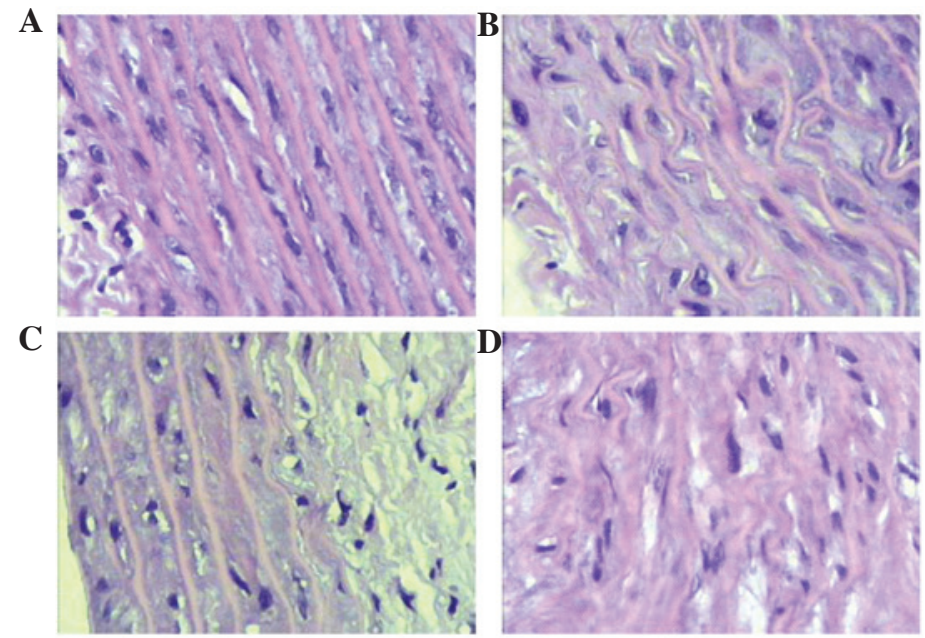

Figure 3. Testosterone induces significant reductions in necrosis and mucoid degeneration in the thoracic aorta tissues. (A) Control group; (B) high-fat-diet + castration group; (C) high-fat-diet + castration + low-dose testosterone group; (D) high-fat-diet + castration + high-dose testosterone group. Hematoxylin and eosin staining (magnification, $\mathrm{x} 400$ ).
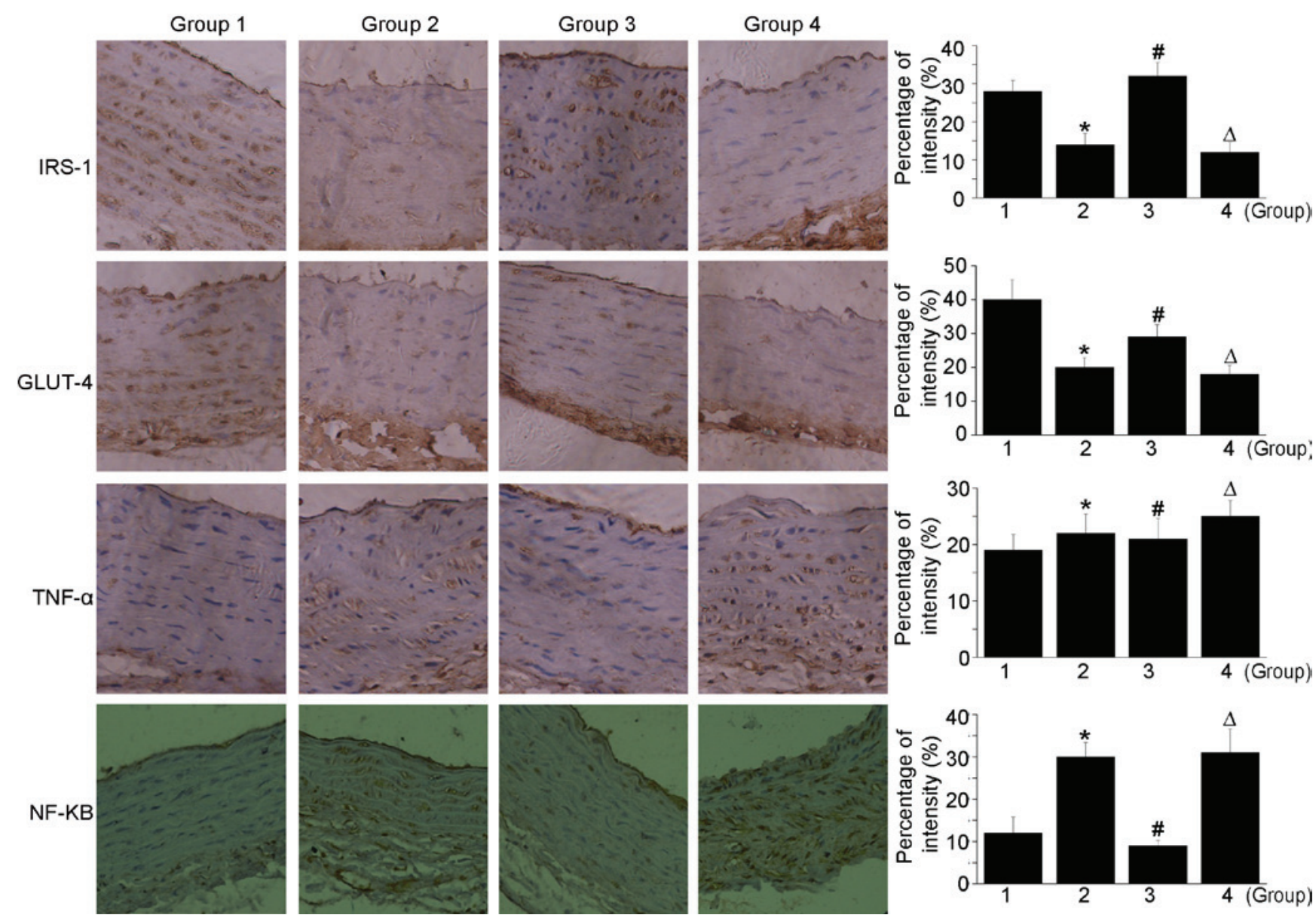

Figure 4. Immunohistochemical staining of sections obtained from the thoracic aorta. High doses of testosterone further increase the expression levels of TNF- $\alpha$ and NF- $\kappa$ B. In the castration + low-dose testosterone group, there were decreases in TNF- $\alpha$ and NF- $\kappa$ B staining, compared with the castration + high-fat diet group. Magnification, $\mathrm{x} 200{ }^{*} \mathrm{P}<0.05$, group 2 compared with group $1 ;{ }^{*} \mathrm{P}<0.05$, group 3 compared with group $2,{ }^{\wedge} \mathrm{P}<0.05$, group 4 compared with group 2 . Group 1, control; group 2, high-fat-diet + castration; group 3; high-fat-diet + castration + low-dose testosterone group 4, high-fat-diet + castration + high-dose testosterone; IRS-1, insulin receptor substrate-1; GLUT-4, glucose transporter type 4; TNF- $\alpha$ tumor necrosis factor- $\alpha$; NF- $\mathrm{kB}$, nuclear factor- $\kappa \mathrm{B}$.

noted in these parameters as plasma testosterone in group 4 increased. Plasma HDL increased as plasma testosterone in group 2 increased. Plasma HDL increased as plasma testosterone in group 4 increased.
Morphological changes in thoracic aorta tissues. As shown in Fig. 3, the inner elastic lamina and endothelial cells in the thoracic aorta tissues were intact in the control group, with no necrosis or mucoid degeneration (Fig. 3A). However, an 

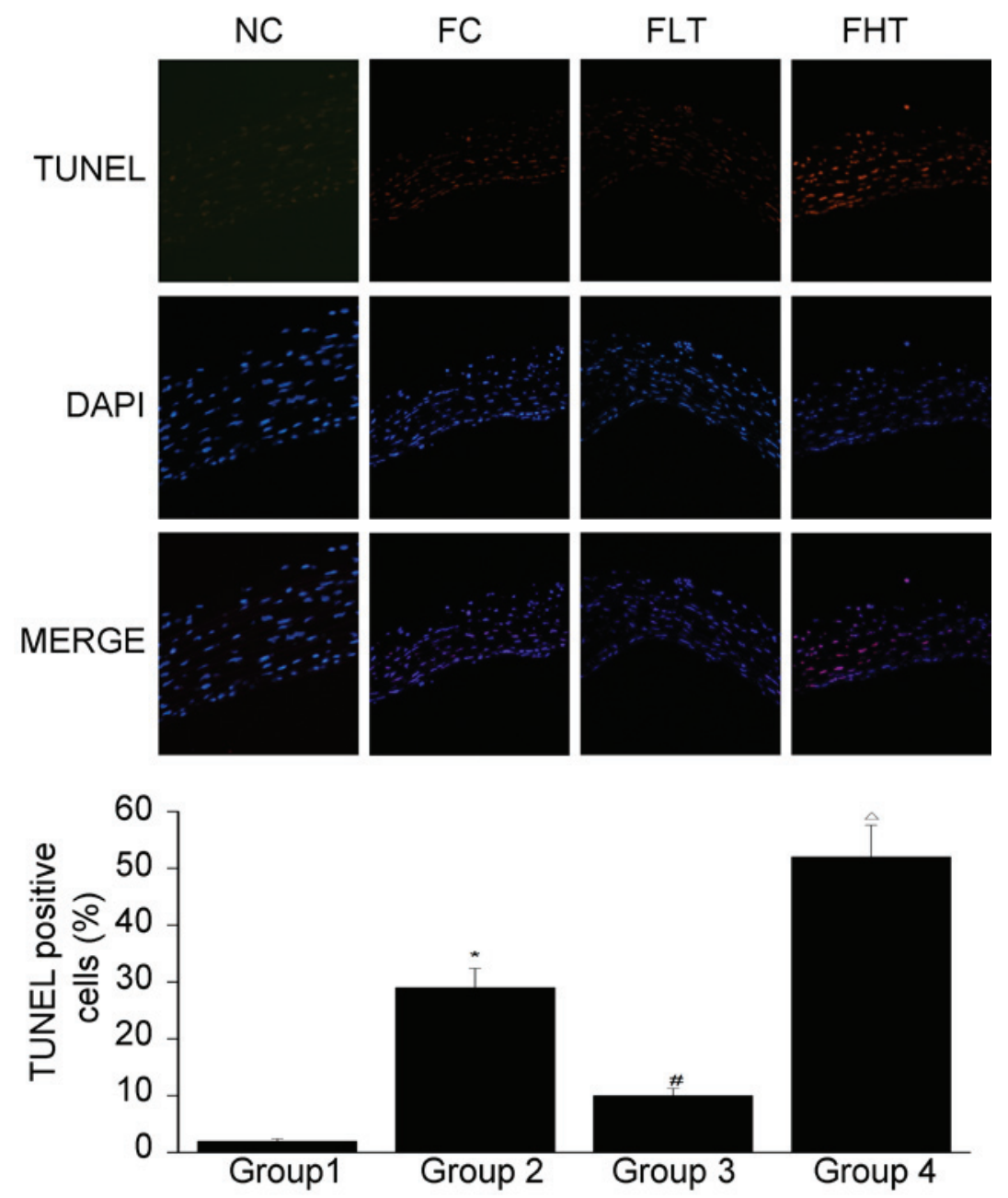

Figure 5. Low doses of testosterone induce lower levels of apoptosis, compared with the castration group. High doses of testosterone resulted in increased cellular apoptosis. The data are presented as the mean \pm standard deviation, based on three independent experiments. "P $<0.05$, group 2 compared with group $1 ;{ }^{*} \mathrm{P}<0.05$, group 3 compared with group $2,{ }^{\Delta} \mathrm{P}<0.05$, group 4 compared with group 2 . Group 1 , control; group 2 , high-fat-diet + castration; group 3 ; high-fat-diet + castration + low-dose testosterone group 4 , high-fat-diet + castration + high-dose testosterone.

irregular lining, with partial endothelial cell loss, necrosis and mucoid degeneration was observed in group 2, together with wrinkling and deformation of the elastic lamina (Fig. 3B). In group 3, a significant reduction was observed in necrosis and mucoid degeneration, compared with group 2 (Fig. 3C). However, in the thoracic aorta tissues obtained from rats treated with high-dose testosterone replacement, severe necrosis and mucoid degeneration were noted, together with deformation of the elastic lamina and endothelium (Fig. 3D).

Immunohistochemical staining. Almost no staining of IRS-1 or GLUT-4 were detected in group 2 in the immunohistochemical analysis. However, the expression levels of IRS-1 and GLUT-4 were marked in group 3. Of note, the expression of IRS-1 in group 3 was significantly higher, compared with that in the normal group. In group 4, the lowest levels of IRS-1 and GLUT-4 were detected, as shown in Fig. 4. Castration in combination with a high-fat diet contributed to increases in the expression levels of TNF- $\alpha$ and NF- $\kappa \mathrm{B}$. In addition, treatment with a high dose of testosterone further increased the expression levels of $\mathrm{TNF}-\alpha$ and $\mathrm{NF}-\kappa \mathrm{B}$. In the castration + low-dose testosterone group, there was a decrease in TNF- $\alpha$ and NF- $\mathrm{BB}$ staining, compared with the castration + high-fat diet group.

TUNEL staining. The results of the TUNEL staining are shown in Fig. 5. Compared with group 1, a significant increase was observed in cell death in group 2 . In addition, a significant decrease in cell death was observed in group 3, compared with group 2. By contrast, a significant increase in cell death was observed in group 4, compared with group 2. This indicated that testosterone at a physiological dose attenuated cellular apoptosis, whereas a high dose of testosterone aggravated the apoptosis.

Western blot analysis. As shown in Fig. 6, the protein levels of IRS-1, AKT and GLUT-4 in group 2 were lower, compared with the levels in group 1. Following exposure to low-dose testosterone, the protein expression levels of IRS-1, AKT and GLUT-4 in group 3 were markedly increased, compared with group 2. However, their levels of expression remained lower, compared with the normal control (group 1). By contrast, exposure to a high dose of testosterone resulted in significant decreases in the levels of IRS-1, AKT and GLUT-4 in group 4, compared with those 

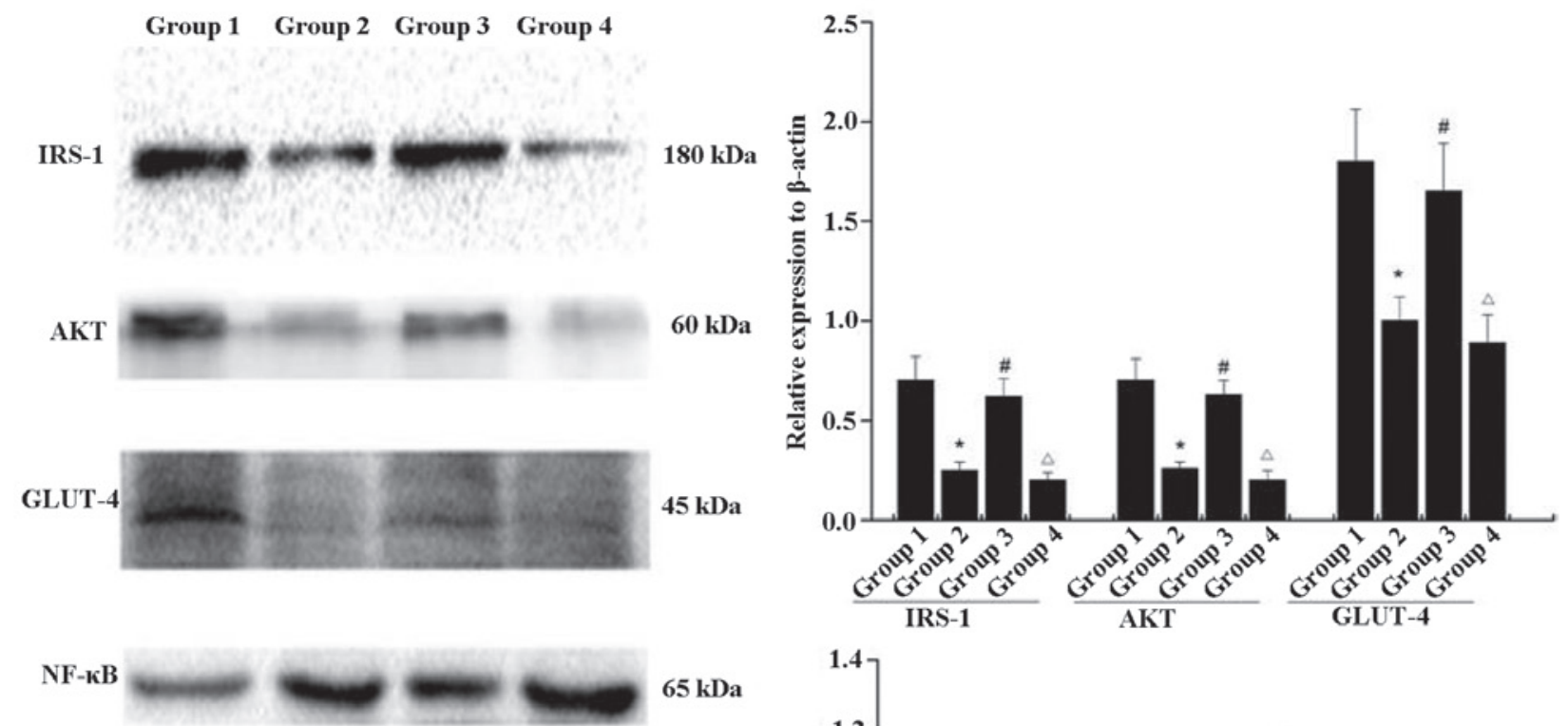

TNF- $\alpha$

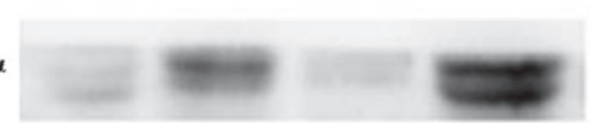

PI3K

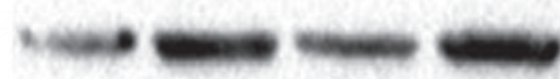

$\beta$-actin

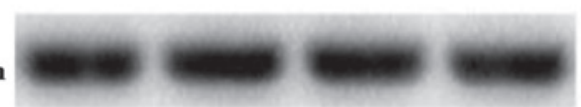

$65 \mathrm{kDa}$

$25 \mathrm{kDa}$

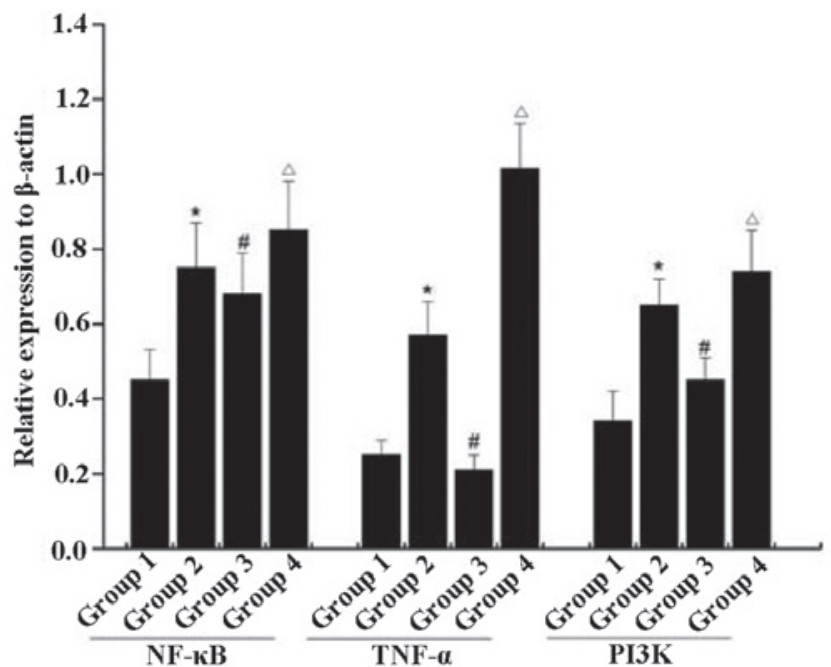

Figure 6. Low-dose testosterone upregulates the expression levels of IRS-1, AKT, GLUT-4 protein, NF-кB, TNF- $\alpha$ and PI3K, compared with those of animals on a high-fat diet following castration. High doses of testosterone resulted in a significant decrease in the levels of IRS-1, AKT, GLUT-4, NF- $k B$, TNF- $\alpha$ and PI3K, compared with animals fed a high-fat diet following castration. Error bars represent the standard deviation. "P<0.05, group 2 compared with group 1; " $\mathrm{P}<0.05$, group 3 compared with group 2 ; ${ }^{\wedge} \mathrm{P}<0.05$, group 4 compared with group 2 . Group 1, control; group 2, high-fat-diet + castration; group 3 ; high-fat-diet + castration + low-dose testosterone; group 4, high-fat-diet + castration + high-dose testosterone; IRS-1, insulin receptor substrate-1; GLUT-4, glucose transporter type 4; TNF- $\alpha$ tumor necrosis factor- $\alpha$; NF- $\mathrm{kB}$, nuclear factor- $\mathrm{\kappa B}$; PI3K, phosphoinositide 3-kinase.

A

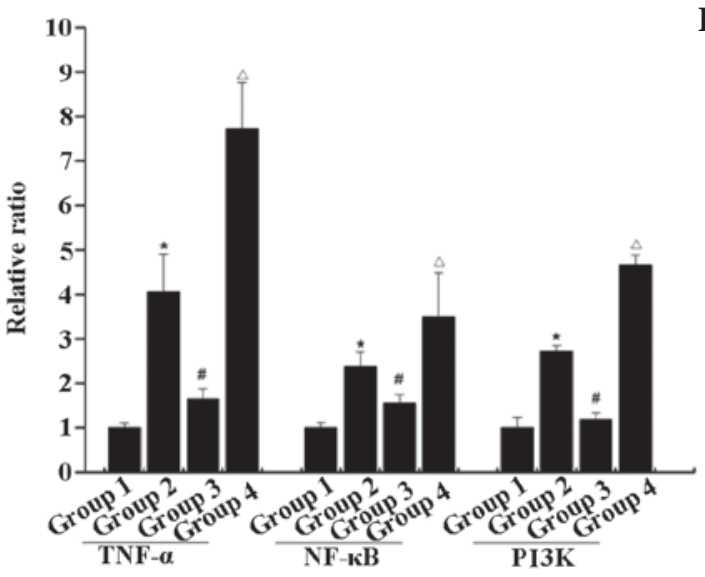

B

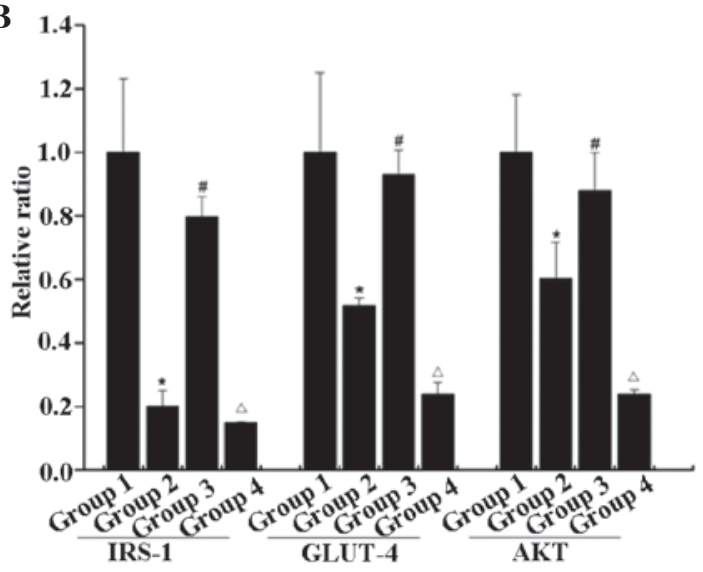

Figure 7. mRNA levels of (A) NF- $\mathrm{kB}$, TNF- $\alpha$ and PI3K, and (B) IRS-1, AKT and GLUT-4. Compared with the rats subjected to castration and fed a high-fat diet, low-dose testosterone induced upregulation of the mRNA levels of IRS-1, AKT and GLUT-4, and downregulation of the mRNA levels of NF-кB, TNF- $\alpha$ and PI3K. However, high-dose testosterone resulted in a significant decrease in the levels of IRS-1, AKT and GLUT-4, and a significant increase in the mRNA levels of NF-кB, TNF- $\alpha$ and PI3K, compared with the low dose group. Error bars represent the standard deviation. ${ }^{*} \mathrm{P}<0.05$, group 2 compared with group $1 ;{ }^{*} \mathrm{P}<0.05$, group 3 compared with group $2 ;{ }^{\circ} \mathrm{P}<0.05$, group 4 compared with group 2 . Group 1 , control; group 2 , high-fat-diet + castration; group 3; high-fat-diet + castration + low-dose testosterone group 4, high-fat-diet + castration + high-dose testosterone; IRS-1, insulin receptor substrate-1; GLUT-4,

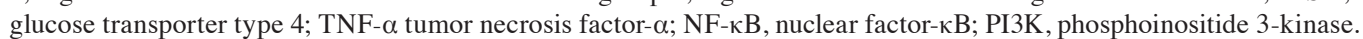


in group 2. In addition, significant upregulation in the levels of $\mathrm{NF}-\kappa \mathrm{B}, \mathrm{TNF}-\alpha$ and PI3K were observed in group 2, compared with the normal control. High-dose testosterone caused further increases in the levels of NF- $\kappa \mathrm{B}, \mathrm{TNF}-\alpha$ and PI3K, compared with those in group 2 , and a low dose of testosterone caused decreases in the levels of NF- $\kappa \mathrm{B}, \mathrm{TNF}-\alpha$ and PI3K in group 3 , compared with group 2. However, the levels of NF- $\mathrm{KB}$ and PI3K in group 3 remained higher than in the normal control.

$R T$ - $q P C R$. As shown in Fig. 7, the mRNA expression levels of IRS-1, AKT and GIUT-4 in group 2 were lower than those in the normal control. Following exposure to low-dose testosterone, the mRNA expression levels of IRS-1, AKT and GLUT-4 in group 3 were markedly increased, compared with those in group 2. However, the mRNA levels of IRS-1, AKT and GLUT-4 in group 3 remained lower than those in the normal control group. Exposure to a high dose of testosterone resulted in significant decreases in the mRNA levels of IRS-1, AKT and GLUT-4 in group 4, compared with group 3. Furthermore, upregulation in the mRNA levels of NF- $\kappa \mathrm{B}, \mathrm{TNF}-\alpha$ and PI3K were observed in group 2, compared with the normal control. A further increase was observed in the mRNA expression levels of $\mathrm{NF}-\kappa \mathrm{B}, \mathrm{TNF}-\alpha$ and $\mathrm{PI} 3 \mathrm{~K}$ following exposure to high-dose testosterone, compared with group 2. Whereas, low-dose testosterone treatment caused a decrease in the mRNA levels of NF- $\kappa \mathrm{B}, \mathrm{TNF}-\alpha$ and PI3K mRNA in group 3. This indicated that the loss of testosterone induced disorder of the PI3K signaling pathway, and contributed to inflammation. A low dose of testosterone may alleviate disorders of PI3K and inflammation, whereas, a high dose of testosterone may aggravate insulin resistance and inflammation.

\section{Discussion}

Testosterone, the most abundant androgen in males, is synthesized and secreted by leydig cells and regulated by pituitary-derived luteinizing hormone (15). To date, testosterone has been used in clinical practice due to its biological properties in the maintenance of normal sexual differentiation, puberty development and anabolism (16). In the present study, the roles of testosterone in glucolipid metabolism and cardiovascular diseases were investigated.

In the early stage of puberty, testosterone is regularly secreted by the testis under the stimulation of luteinizing hormone. The association between endogenous androgen and lipids is complex as it involves various factors, including as age, gender, body mass index and waist-hip ratio. For example, Botella-Carretero et al found that the plasma levels of testosterone of obese patients were higher following bariatric surgery, compared with baseline levels (17). In addition, a linear trend towards lower TC, low triglycerides and higher HDL-C have been reported with the increase of serum testosterone (18). However, other studies have shown that testosterone replacement induces a decrease in plasma HDL and an increase in TC $(19,20)$. In the present study, testosterone was negatively correlated with TC and non-HDL, and was positively correlated with HDL. Adverse effects were observe from the supraphysiological testosterone concentration on plasma lipids. On this basis, an appropriate dose of testosterone is required in clinical practice.
Previously, testosterone was considered to be associated with the occurrence of coronary heart disease (21). However, recent data has revealed that testosterone is negatively correlated with the occurrence of coronary heart disease (22). In addition, testosterone is important in anti-atherosclerosis. It is known that the vascular endothelium is the barrier between circulating blood and vascular smooth muscle, and it is the major component of endocrine organs. It has been reported to be involved in the synthesis and secretion of several vasoactive substances associated with the regulation of vasomotor function. Testosterone can promote the secretion of nitric oxide from vascular endothelial cells, which affects vasomotor function (23). The results of the present study revealed that testosterone alleviated injury to the morphology of the thoracic aorta induced by hypoandrogenism.

Increasing attention has been focussed on the roles of insulin resistance in metabolic syndrome (22). For example, epidemiological data indicates that the incidence of type 2 diabetes in males is higher than in females, which may be affected by endogenous hormones (24). Increasing evidence has revealed that hypotestosteronemia is associated with increased risks of developing metabolic syndrome and diabetes, as well as insulin resistance (25). Previous studies have shown that testosterone replacement can ameliorate the pathological components of metabolism syndrome, however, adverse effects may occur in the presence of excessive administration $(26,27)$. To investigate the potential mechanism underlying how testosterone is involved in insulin resistance, the present study investigated the activity of PI3K/AKT, a major component in the insulin signaling pathway for glucose transport. In the present study, rats subjected to castration exhibited upregulated expression of PI3K and downregulated expression levels of IRS-1, GLUT-4 and AKT. In the animals treated with testosterone, the levels of IRS-1, GLUT-4 and AKT were upregulated, together with downregulation in the levels of NF- $\mathrm{BB}$ and TNF- $\alpha$. On this basis, the present study hypothesized that testosterone may regulate glucolipid metabolism through modulation of the PI3K signaling pathway.

In conclusion, the present study demonstrated that castration induced marked disorder of glucolipid metabolism and vascular injuries in male pubescent rats. Low-dose testosterone replacement treatment ameliorated the damage caused by castration via the PI3K/AKT signaling pathway. However, high-dose testosterone induced severe adverse effects, indicating an appropriate dose of testosterone is necessary. Testosterone deficiency and overdose induced disorder of glucolipid metabolism and vascular injuries in male pubescent rats. Thus, the dosage of testosterone used must be appropriate for the treatment of patients with testosterone deficiency and testosterone overdose should be avoided.

\section{Acknowledgements}

This study was supported by The Neurological Institute of Tianjin Medical University General Hospital and the Tianjin High Education Development Fund (grant no. 20140126).

\section{References}

1. Karoutsou E and Polymeris A: Environmental endocrine disruptors and obesity. Endocr Regul 46: 37-46, 2012. 
2. Boodai SA, Cherry LM, Sattar NA and Reilly JJ: Prevalence of cardiometabolic risk factors and metabolic syndrome in obese Kuwaiti adolescents. Diabetes Metab Syndr Obes 7: 505-511, 2014.

3. Kursawe R and Santoro N: Metabolic syndrome in pediatrics. Adv Clin Chem 65: 91-142, 2014.

4. Özer S, Yılmaz R, Özlem Kazancı N, Sönmezgöz E, Karaaslan E, Altuntaş B and Emre Kuyucu Y: Higher HDL levels are a preventive factor for metabolic syndrome in obese Turkish children. Nutr Hosp 31: 307-312, 2014.

5. Denzer C, Thiere D, Muche R, Koenig W, Mayer H, Kratzer W and Wabitsch M: Gender-specific prevalences of fatty liver in obese children and adolescents: Roles of body fat distribution sex steroids and insulin resistance. J Clin Endocrinol Metab 94: 3872-3881, 2009.

6. Shelton JB and Rajfer J: Androgen deficiency in aging and metabolically challenged men. Urol Clin North Am 39: 63-75, 2012.

7. Dhindsa S, Prabhakar S, Sethi M, Bandyopadhyay A, Chaudhuri A and Dandona P: Frequent occurrence of hypogonadotropic hypogonadism in type 2 diabetes. J Clin Endocrinol Metab 89: 5462-5468, 2004.

8. Moriarty-Kelsey M, Harwood JE, Travers SH, Zeitler PS and Nadeau KJ: Testosterone, obesity and insulin resistance in young males: Evidence for an association between gonadal dysfunction and insulin resistance during puberty. J Pediatr Endocrinol Metab 23: 1281-1287, 2010.

9. Janjgava S, Zerekidze T, Uchava L, Giorgadze E and Asatiani K Influence of testosterone replacement therapy on metabolic disorders in male patients with type 2 diabetes mellitus and androgen deficiency. Eur J Med Res 19: 56, 2014.

10. Sattler F, He J, Chukwuneke J, Kim H, Stewart Y, Colletti P, Yarasheski $\mathrm{K}$ and Buchanan T: Testosterone supplementation improves carbohydrate and lipid metabolism in some older men with abdominal obesity. J Gerontol Geriatr Res 3: 1000159, 2014.

11. Yeap BB, Alfonso H, Chubb SA, Handelsman DJ, Hankey GJ, Golledge J, Flicker L and Norman PE: Lower plasma testosterone or dihydrotestosterone, but not estradiol, is associated with symptoms of intermittent claudication in older men. Clin Endocrinol (Oxf) 79: 725-732, 2013.

12. Srinath R, Hill Golden S, Carson KA and Dobs A: Endogenous testosterone and its relationship to preclinical and clinica measures of cardiovascular disease in the atherosclerosis risk in communities (ARIC) study. J Clin Endocrinol Metab 100: $1602-1608,2015$

13. Yu J, Akishita M, Eto M, Ogawa S, Son BK, Kato S, Ouchi Y and Okabe T: Androgen receptor-dependent activation of endothelial nitric oxide synthase in vascular endothelial cells: Role of phosphatidylinositol 3-kinase/akt pathway. Endocrinology 151 $1822-1828,2010$.

14. Li F, Li L, Zhong Y, Xie Q, Huang J, Kang X, Wang D, Xu L and Huang T: Relationship between LTR methylation and gag expression of HIV-1 in human spermatozoa and sperm-derived embryos. PLoS One 8: e54801, 2013.
15. Beaudin G: Men and low testosterone. Diabetes Self Manag 32: 24-27, 2015

16. Jockenhövel F: Testosterone therapy - what, when and to whom? Aging Male 7: 319-324, 2004.

17. Botella-Carretero JI, Balsa JA, Gómez-Martin JM, Peromingo R, Huerta L, Carrasco M, Arrieta F, Zamarron I, Martin-Hidalgo A and Vazquez C: Circulating free testosterone in obese men after bariatric surgery increases in parallel with insulin sensitivity. J Endocrinol Invest 36: 227-232, 2013.

18. Zhang N, Zhang H, Zhang X, Zhang B, Wang F, Wang C, Zhao M, Yu C, Gao L, Zhao J and Guan Q: The relationship between endogenous testosterone and lipid profile in middle-aged and elderly Chinese men. Eur J Endocrinol 170: 487-494, 2014

19. Gu YQ, Wang XH, Xu D, Peng L, Cheng LF, Huang MK, Huang ZJ and Zhang Y: A multicenter contraceptive efficacy study of injectable testosterone undecanoate in healthy Chinese men. J Clin Endocrinol Metab 88: 562-568, 2003.

20. Gårevik N, Rane A, Björkhem-Bergman L and Ekström L: Effects of different doses of testosterone on gonadotropins, 25-hydroxyvitamin D3 and blood lipids in healthy men. Subst Abuse Rehabil 5: 121-127, 2014.

21. Rosano GM, Sheiban I, Massaro R, Pagnotta P, Marazzi G, Vitale C, Mercuro G, Volterrani M, Aversa A and Fini M: Low testosterone levels are associated with coronary artery disease in male patients with angina. Int J Impot Res 19: 176-182, 2007.

22. Shores MM, Matsumoto AM, Sloan KL and Kivlahan DR: Low serum testosterone and mortality in male veterans. Arch Intern Med 166: 1660-1665, 2006.

23. Duckles SP and Miller VM: Hormonal modulation of endothelial NO production. Pflugers Arch 459: 841-851, 2010.

24. Lapauw B, Ouwens M, 't Hart LM, Wuyts B, Holst JJ, T'Sjoen G, Kaufman JM and Ruige JB: Sex steroids affect triglyceride handling, glucose-dependent insulinotropic polypeptide and insulin sensitivity: A 1-week randomized clinical trial in healthy young men. Diabetes Care 33: 1831-1833, 2010.

25. Kupelian V, Page ST, Araujo AB, Travison TG, Bremner WJ and McKinlay JB: Low sex hormone-binding globulin, total testosterone and symptomatic androgen deficiency are associated with development of the metabolic syndrome in nonobese men. J Clin Endocrinol Metab 91: 843-850, 2006.

26. Jones TH, Arver S, Behre HM, Buvat J, Meuleman E, Moncada I, Morales AM, Volterrani M, Yellowlees A, Howell JD and Channer KS; TIMES2 Investigators: Testosterone replacement in hypogonadal men with type 2 diabetes and/or metabolic syndrome (the TIMES2 study). Diabetes Care 34: 828-837, 2011.

27. Guay AT: The emerging link between hypogonadism and metabolic syndrome. J Androl 30: 370-376, 2009. 